\title{
An In-Silico Study Into the Impact of Electrophysiological Variability at the Cellular Level on the Re-entry Patterns in Atrial Fibrillation
}

\author{
Jordan Elliott $^{1}$, Olaf Dössel ${ }^{2}$, Axel Loewe ${ }^{2}$, Luca Mainardi ${ }^{1}$, Valentina Corino ${ }^{1}$, José Felix Rodriguez \\ Matas $^{1}$ \\ ${ }^{1}$ Politecnico Di Milano, Milano, Italy \\ ${ }^{2}$ Karlsruher Institut für Technologie, Karlsruher, Germany
}

\begin{abstract}
Modelling the atria in-silico has become an important method in understanding atrial behaviour. Atrial models typically include regional electrophysiological variability, but neglect cellular variability. The aim of the study is to determine the impact of cellular electrophysiological variability on ectopic beats. Using a population of models approach to introduce regional and cellular variability into the atrial model, ectopic beats were initiated in two locations. Six ectopic beats were applied at a BCL of 130-160ms. The variable model was compared with an equivalent regional homogenous model. Using consistent tissue CV between models, in both the healthy and $A F$ remodeled cases the average model total activation time was later than the variable model (a delay of $26 \mathrm{~ms}$ and $14 \mathrm{~ms}$ respectively). After matching activation times, repolarization was later in the average than the variable models. Latest APD90 in the AF remodeled cases were $268 \mathrm{~ms}$ for the average and 256ms in the variable model. This resulted in a difference in propagation of the ectopic beat. In conclusion, cellular variability has a significant impact on both the depolarization and repolarization phases in the atria for the healthy and AF cases.
\end{abstract}

\section{Introduction}

In silico modelling is often used to model the behaviour of the atria during atrial fibrillation in order to better understand the mechanisms and therefore improve treatment methods. Much of the modelling associated with AF research focusses on the anatomical variability between patients to determine susceptibility. The research investigating the impact of electrophysiology within the atria is limited by the complexity of the models, whereby variability is included on a regional basis ${ }^{[4]}$ and cell-to-cell variability within each atrial region is neglected. Experimental investigations into the cellular variability within atrial regions shows significant levels of cellular variability within the same atria region ${ }^{[1][5][6][9][12]}$.

When modelling the atrial response to AF it is important to have as accurate a model as possible in order to obtain realistic results. It is typically assumed that cellular coupling masks the variability within atrial regions and therefore a regionally homogenous model is used to predict atrial behaviour. But how much of an impact does cellular variability have on the electrophysiological behaviour of the atria? It is the purpose of this study to determine the impact of cellular variability on the atrial electrophysiological response to both sinus rhythm and the presence of ectopic beats.

\section{Methods}

\subsection{Cellular model}

A total of 9 maximum channel conductances were varied $+/-100 \%$ using the Monte Carlo Sampling Method to create a population of models using the Maleckar cellular model for cardiomyocytes ${ }^{[7]}$. Each cellular model was stimulated a $1 \mathrm{~Hz}$ for 10 minutes. The ultimate action potential was used for classification. Any unstable or nonphysiological action potentials were discarded.

Using experimental data to define regional characteristics using 5 biomarkers (Table 1), the population of models was divided into regional populations ${ }^{[1][5][6][9][12]}$. Due to a lack of experimental data regarding the biomarkers for regional tissue in an $\mathrm{AF}$ remodeled atria, the AF remodeled populations were created by applying the percentage changes to 5 channel conductances $^{[8]}$, shown in Table 2 . Again, any unstable or non-physiological action potentials were discarded.

\subsection{Atrial model}


Table 1 Mean and standard deviation of regional biomarkers used for population calibration in the healthy atrial model

\begin{tabular}{l|llllllll}
\multicolumn{2}{c}{$R A$} & $R A A$ & $L A$ & $L A A$ & $A V R$ & $C T / B B r a$ & $B B l a$ & $P M$ \\
\hline$R M P$ & $-75+/-12$ & $-76+/-6.6$ & $-75+/-5.4$ & $-71+/-6.6$ & $-71+/-1.4$ & $-74+/-1.9$ & $-74+/-1.9$ & $-73+/-12$ \\
$A P A$ & $109+/-14$ & $116+/-19$ & $95+/-3.9$ & $120+/-19$ & $119+/-21$ & $126+/-19$ & $116+/-19$ & $123+/-16$ \\
APD20 & $7+/-6.6$ & $7+/-6.6$ & $7+/-6.6$ & $7+/-6.6$ & $7+/-6.6$ & $7+/-6.6$ & $7+/-6.6$ & $7+/-6.6$ \\
APD50 & $95+/-37$ & $139+/-36$ & $72+/-17$ & $118+/-13$ & $50+/-21$ & $157+/-32$ & $124+/-32$ & $98+/-17$ \\
APD90 & $295+/-62$ & $280+/-22$ & $256+/-34$ & $236+/-22$ & $250+/-29$ & $322+/-64$ & $253+/-32$ & $254+/-19$
\end{tabular}

For each regional population, the action potential representing the mean characteristics of the population was assigned to each node associated with that region in the atrial model. This created the regionally homogenous model used for comparison, shown in Figure 1.

Using the regional populations, each node within the whole atrial model was randomly assigned a single cellular model from the associated population with a uniform distribution. This resulted in four comparable models: healthy average atria, healthy variable atria, AF remodeled average atria, and AF remodeled variable atria. Tissue conduction velocity for the healthy atria models were calibrated using the variable model to adjust

Using a total activation time within the observed physiological range for a healthy atria, the tissue conduction velocity was adjusted until the variable healthy model total activation time fell within this accepted range.

For the AF remodeled atria, tissue conduction velocity was reduced by $15 \%$ from the healthy atria CV [8]. The average models were initially simulated using the same conduction velocity as the respective variable models and further adjusted until the total activation times were comparable with the variable models.

Table 2 Percentage changes applied to left and right atrial regions for $A F$ remodeling

\begin{tabular}{|c|c|c|}
\hline & $R A$ regions & LA regions \\
\hline gTo & $-45 \%$ & $-75 \%$ \\
\hline gKur & $-60 \%$ & $-45 \%$ \\
\hline$g K s$ & $+150 \%$ & $+100 \%$ \\
\hline$g K 1$ & $+100 \%$ & $+100 \%$ \\
\hline$g C a L$ & $-65 \%$ & $-65 \%$ \\
\hline
\end{tabular}
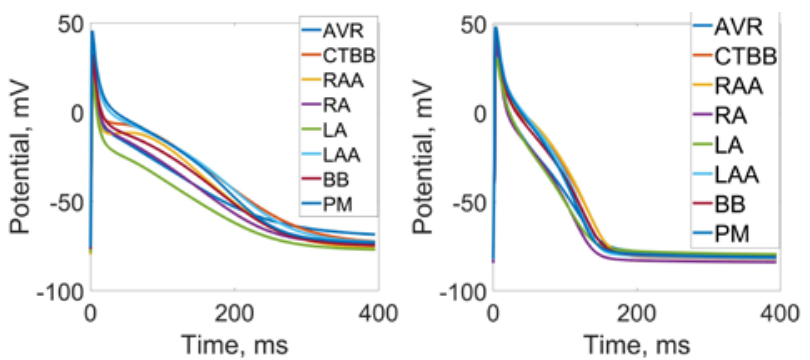

Figure 1 Regional population average models for the healthy atrial regions (Left) and AF remodeled regions (Right) used to create regionally homogenous models.
To stabilise the atrial models, each model was pre-paced using 10 stimuli at a BCL of $800 \mathrm{~ms}$, stimulus duration $2 \mathrm{~ms}$, amplitude $-50 \mathrm{mV}$. After pre-pacing, each model was stimulated using a single SR for comparison during normal atrial behaviour.

To determine the impact of cellular variability during AF, ectopic beats were applied in two regions: the right and left pulmonary vein ostium ${ }^{[10]}$. SR stimulation was continued throughout ectopic beat stimulation. A total of 719 and 791 nodes were stimulated in the LPVo and RPVo respectively. Six ectopic beats were applied at a BCL ranging from $130-150 \mathrm{~ms}$, stimulus duration 2ms, amplitude $-50 \mathrm{mV}$. SR stimuli were applied at a BCL of 800ms, stimulus duration 2ms, amplitude $-50 \mathrm{mV}$.

Table 3 Total activation times for the healthy and AF remodeled cases using the same conduction velocity and an increased conduction velocity in the regionally homogenous case.

\begin{tabular}{l|llll} 
& $\begin{array}{l}\text { Av. } \\
\text { model }\end{array}$ & $\begin{array}{l}\text { Var. } \\
\text { model }\end{array}$ & $\begin{array}{l}\text { Increase } \\
\text { d Av. CV }\end{array}$ & $\begin{array}{l}\text { \%increas } \\
\text { e CV }\end{array}$ \\
\hline $\begin{array}{l}\text { Healthy } \\
\text { AF } \\
\text { remodeled }\end{array}$ & 150 & 124 & 128 & $26 \%$ \\
& 168 & 144 & 144 & $8 \%$
\end{tabular}

\subsection{Analysis}

Models were compared through total activation time, the time at which the latest APD90 was reached in each model, and visual differences in propagation patterns throughout both healthy atrial behaviour and ectopic beat behaviour.

\section{Results}

\subsection{Population variability}

Populations showed a reduction in APD50 and APD90 from the healthy atrial regions to the AF remodeled regions. Figure 2 shows the reduction observed in regional populations for the APD50 and APD90 biomarkers.

\subsection{Healthy atrial behavior}

\subsection{Simulations}




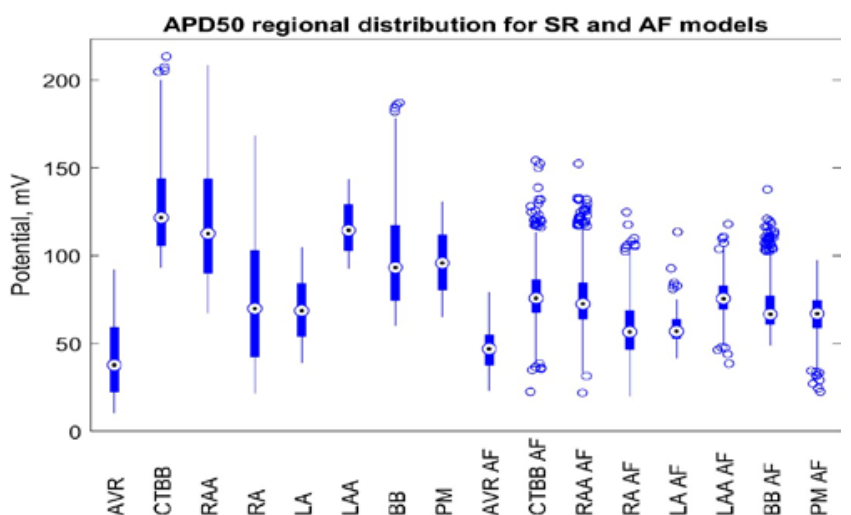

Figure 2 Population distribution of APD50 (left) and APD90 (rig

Using consistent tissue CV between models, in both the healthy and AF remodeled cases the average model total activation time was later than the variable model (a delay of $26 \mathrm{~ms}$ and $14 \mathrm{~ms}$ respectively). Table 3 shows the total activation times for the average and variable models in both the healthy and AF remodeled cases.

The average models were recalibrated to match the variable model TAT. In the healthy model this required a $26 \%$ increase in CV to bring the average TAT within 5\% of the variable TAT. In the AF remodeled case, the CV increase was smaller, requiring an $8 \%$ increase to match TAT between models.

After matching activation times, repolarization was later in the average than the variable models. Latest APD90 in the AF remodeled cases were $268 \mathrm{~ms}$ for the average and $256 \mathrm{~ms}$ in the variable model. Additionally, in both the healthy and AF remodeled cases, small differences in propagation patterns were observed.

Additionally, in both the healthy and AF remodeled cases, small differences in propagation patterns were observed. In both the healthy and AF remodeled cases the variable model propagation had a faster propagation with different morphology from initial stimulation. This can be seen in Figure 3 whereby the left shows the average model propagation and the right shows the variable propagation

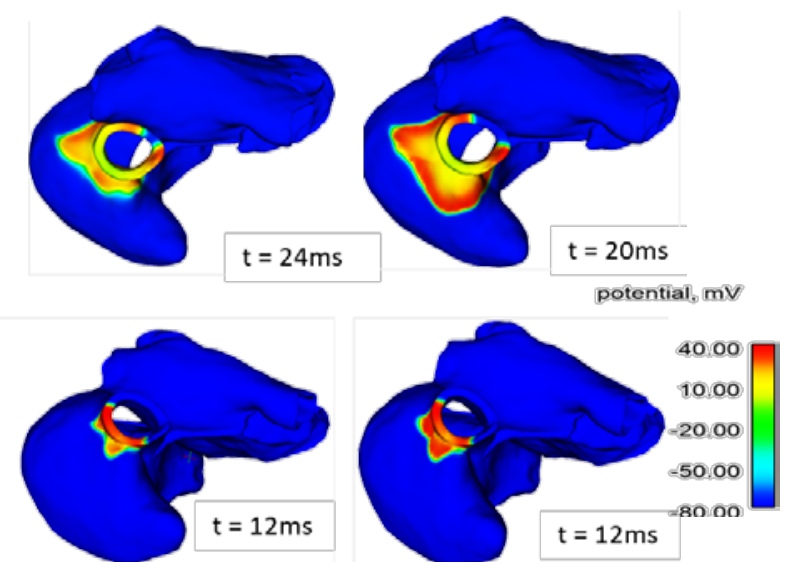

Figure 3 Sinus rhythm propagation in healthy (top) and AF remodeled (bottom) average (left) and variable (right) models during early stages of propagation

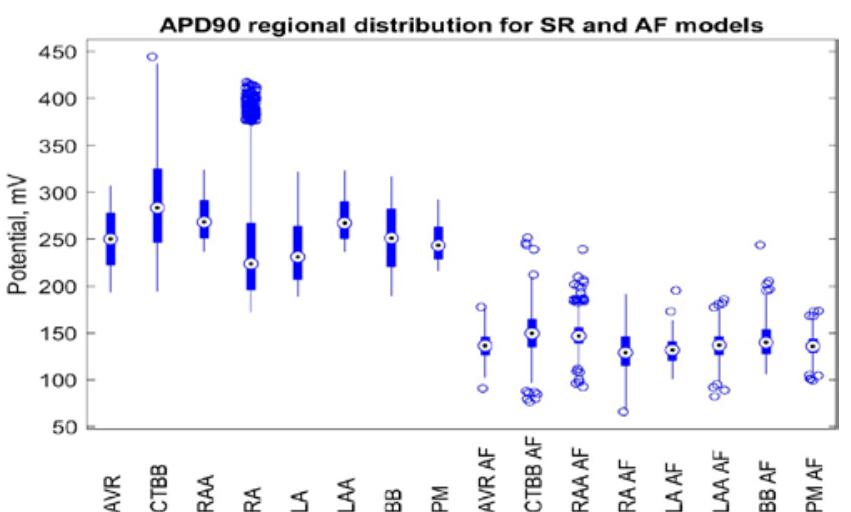

ht) for healthy (first 8) and AF remodeled atria regions (last 8).

for both the healthy (top) and AF remodeled (bottom) atria. This results in a different propagation across the right atria as shown in Figure 4 at t $=88 \mathrm{~ms}$ in the average model and $\mathrm{t}=80 \mathrm{~ms}$ in the variable model. The black boxes highlight regions in which the propagation differs between models.
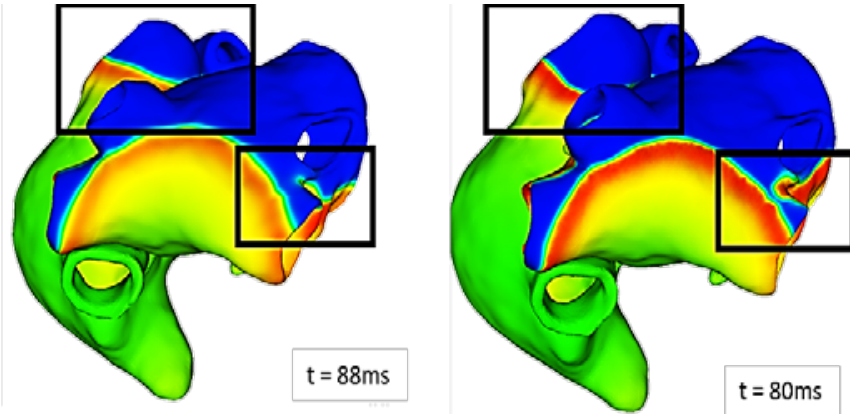

Figure 4 Later sinus rhythm propagation in average model with increased CV (left) and variable (right) healthy atrial models. Black boxes show regions where propagation differs between average and variable models

\subsection{AF remodeled atria ectopic beats}

Figure 5 shows the propagation of a RPV ectopic beat across the variable (top) and average (bottom) AF remodeled As shown in figure 5, the variable model propagates through into the right atria through the coronary sinus whereas the same ectopic beat is blocked in the average model. This results in a different wavefront morphology and therefore a different pattern of repolarization. This is likely due to the difference in repolarization times between the average and variable AF remodeled atria.

Similarly, the difference in repolarization times between the average and variable AF remodeled atria resulted in an ectopic beat propagating in the variable model while failing to propagate in the average model. These differences observed as a result of ectopic beats could lead to differences in behavior during reentries in AF. Similar differences between models were observed in the LPV ectopic beat simulations.

\section{Conclusion}




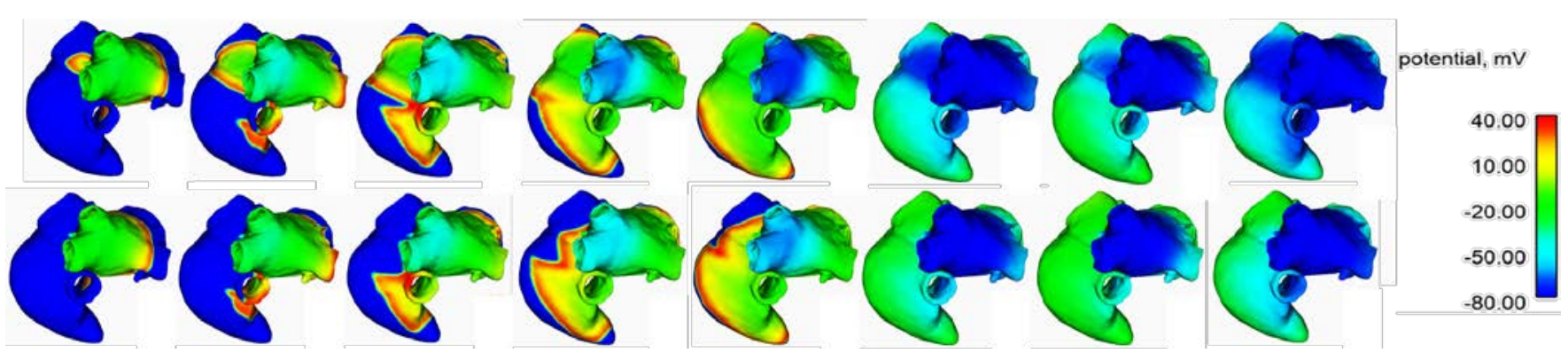

Figure 5 Propagation of RPV ectopic beat in the variable AF remodeled atria (top) and the average AF remodeled atria (bottom), from left to right $t=456 \mathrm{~ms}, 480 \mathrm{~ms}, 492 \mathrm{~ms}, 504 \mathrm{~ms}, 516 \mathrm{~ms}$, 580ms, 568ms, $592 \mathrm{~ms}$.

In conclusion the inclusion of cellular variability in atrial modelling results in a need for a reduction in tissue conduction velocity to maintain physiological total activation times observed in regionally homogenous models. In both the healthy and AF remodeled cases, using comparable total activation times results in small differences in wavefront morphology between the average and variable models.

Additionally, in both the SR and AF remodeled cases, the repolarization across the atria was slower in the average model than the variable model. This shows that even with accounting for the increased propagation velocity across the variable model, the behaviour in depolarisation differs significantly compared with the regionally homogenous model. When applying ectopic beats to the average and variable models with comparable total activation times, differences in propagation patterns are observed to the extent that an ectopic beat that propagates in the variable model fails to propagate in the average model. Similarly, interatrial blocks observed in the average model EB are not present in the variable model EB. This could result in a difference in susceptibility to atrial fibrillation.

\section{Limitations}

These results only observe the difference in propagation resulting from cellular variability using one cellular model. Future work includes using the Courtemanche cellular model $^{[2]}$ and different combinations of cellular variability using the Maleckar model ${ }^{[7]}$. Additionally, the impact of variability on ectopic beats in other locations and on reentry patterns during AF are to be investigated ${ }^{[10]}$.

\section{Acknowledgments}

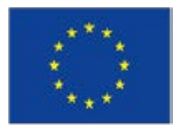

This project has received funding from the European Union's Horizon 2020 research and innovation programme under the Marie Sklodowska-Curie grant agreement No. 766082.

\section{References}

[1] C. Sánchez et al., "Inter-subject variability in human atrial action potential in sinus rhythm versus chronic atrial fibrillation,” PLoS One, vol. 9, no. 8, 2014.

[2] Courtemanche M, Ramirez RJ, Nattel S. "Ionic mechanisms underlying human atrial action potential properties: insights from a mathematical model”. Am J Physiol. 1998;275(1 Pt 2):H301-21, 1998.

[3] D. Li, L. Zhang, J. Kneller, and S. Nattel, "Potential ionic mechanism for repolarization differences between canine right and left atrium," Circ. Res., vol. 88, no. 11, pp. 1168-1175, 2001.

[4] Godoy EJ, Sánchez-Quintana D, Rodríguez JF, Ferrer A, Saiz J, Martínez L, et al. "Detailed Anatomical and Electrophysiological Models of Human Atria and Torso for the Simulation of Atrial Activation”. PLoS One;10(11):e0141573, 2015.

[5] H. Zhang, O. Dössel, G. Seemann, F. B. Sachse, A. V Holden, and C. Höper, "Heterogeneous three-dimensional anatomical and electrophysiological model of human atria," Philos. Trans. R. Soc. A Math. Phys. Eng. Sci., vol. 364, no. 1843, pp. 1465-1481, 2006.

[6] J. Feng, L. Yue, Z. Wang, and S. Nattel, "Ionic mechanisms of regional action potential heterogeneity in the canine right atrium,” Circ. Res., vol. 83, no. 5, pp. 541-551, 1998. [7] M. Maleckar et al., " $\mathrm{K}+$ current changes account for the rate dependence of the action potential in the human atrial myocyte ,” Am. J. Physiol. Circ. Physiol., vol. 297, no. 4, pp. H1398-H1410, 2009.

[8] Martinez-Mateu L, Romero L, Ferrer-Albero A, Sebastian R, Rodríguez Matas JF, Jalife J, et al. "Factors affecting basket catheter detection of real and phantom rotors in the atria: A computational study." PLoS Comput Biol. 2018;14(3):1-26, 2018.

[9] O. V. Aslanidi et al., "3D virtual human atria: A computational platform for studying clinical atrial fibrillation," Prog. Biophys. Mol. Biol., vol. 107, no. 1, pp. 156-168, 2011. [10] Tobón C, Ruíz C, Rodríguez JF, Hornero F, Ferrero JM, Saiz J. "Vulnerability for reentry in a three dimensional model of human atria: A simulation study". Annu Int Conf IEEE Eng Med Biol Soc EMBC'10. 2010;224-7, 2010.

[11] U. Ravens et al., "Balance between sodium and calcium currents underlying chronic atrial fibrillation termination: An in silico intersubject variability study," Hear. Rhythm, vol. 13, no. 12, pp. 2358-2365, 2016.

[12] X. Liu et al., "From ionic to cellular variability in human atrial myocytes: an integrative computational and experimental study,” Am. J. Physiol. Circ. Physiol., vol. 314, no. 5, pp. H895-H916, 2017.

Address for correspondence:

Jordan Elliott

LabS, Piazza Leonardo da Vinci, 32, 20133 Milano MI

Jordan.elliott@polimi.it 